\title{
Study on the Gear Gap of Gun Tower LI Jiaqi ${ }^{1, a}$, LIAO Zili, YUAN Dong, YANG Guibing \\ ${ }^{1} 21$ Court, Dujiakan, Fengtai District, Beijing, P.R. of China \\ alijiaqi8643@163.com
}

Keywords: Gear gap, Gun tower system, Traversing mechanism

\begin{abstract}
The gap of the gears has a huge influence in tank's transmission system even fasten the appendixes'deformation and the motor's damage. In order to study the influence of gear's gap and aimed to the nonlinear characteristic of gear's gap in the transmission, established a 3D model in CREO and put it into ADAMS to simulate. This paper has studied the difference of the force of the transmission in different gear's gap, and the influence of the gap to the transmission. All the work had built a good foundation.
\end{abstract}

\section{Introduction}

The gun system of the tank is a nonlinear and complex system with a strong inner nature. Inside this system, the unsure nonlinear factors such as the gear gap, the elastic deformation and the friction torque, are easily to lead to a series problems like steady state holding shift and drive dead zone. Especially the gear gap, which can lead to a huge change of the forces, and make the deformation of the gear, the trunnion and the toothed sector, would make a huge damage to the gun system.

In order to control the disturbance produced by gear gap, a 3D solid model was established. The gun system was analyzed when the gear gap was changed in different condition so that the study of the dynamic influence of the gap.

\section{Establishment a 3D solid model}

Dynamic study must have an exact 3D solid model. Compared to the traditional mathematic model, the 3D solid model's simulation get closer to the real life.

The base structure of the gun system was like the Fig.1 shows. In real life, the gear gap was a crucial factor which is nonlinear and unsure in this system.

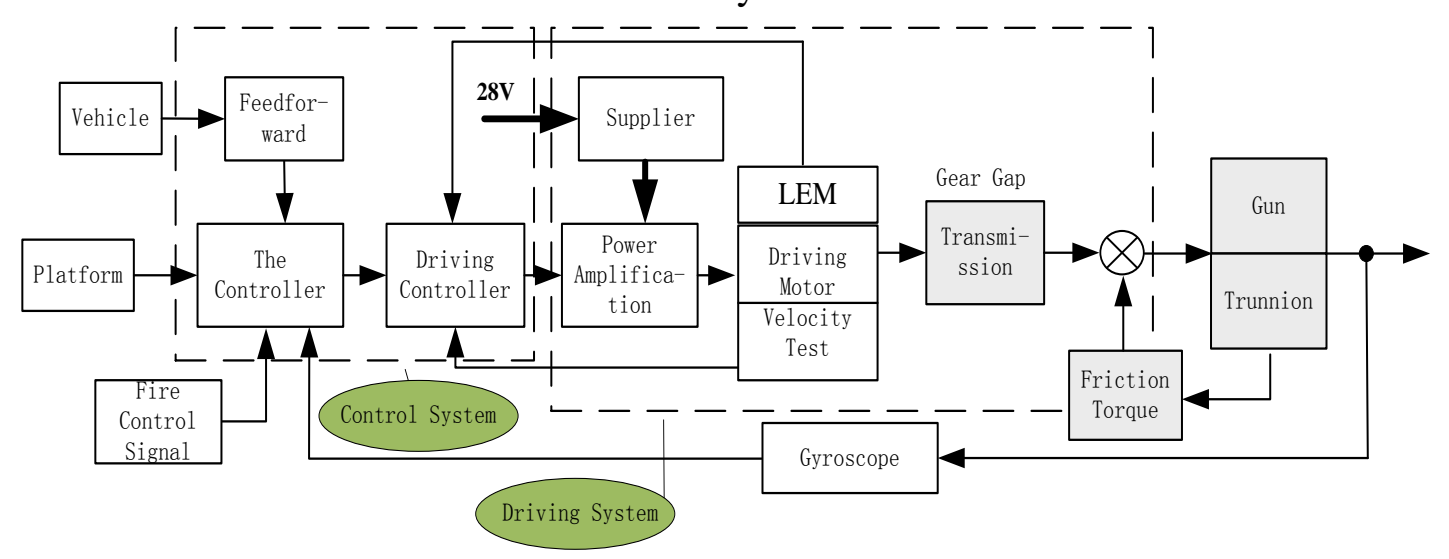

Fig.1 The structure of gun system

According to the structure shown in Fig.1, a dynamic model of gun system was established by the use of CREO and ADAMS. It means that the mathematic model was established in CREO and import into ADAMS to set the joints. The modeling flow was shown as Fig.2. 


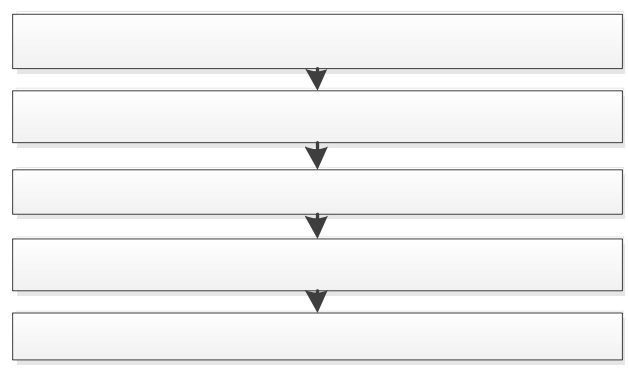

Fig. 2 The flow of modeling

According to a certain kind of armored vehicle, a series of relevant components were established and assembled into a entire gun system. Finally, it was shaped a full model according to Fig.2 and the final model was shown in Fig.3.

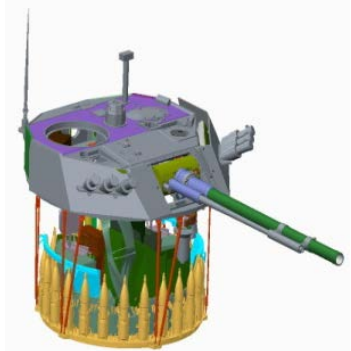

Fig.3 The entire model of gun system

\section{The gear gap}

Compared to the traditional gun system, the all-electric gun system adopted the electric drive system in both the horizontal direction and the elevating direction. In the horizontal direction, the multiple gear system slows down the velocity to meet the gun or the tower's need. The bind-seize should be avoided due to a suitable distance of geared mesh. Hence, there must be a few problems such as the reversing shock and delayed drive time because of the gap. The gun power system would be influenced in a heavy.

Take the traversing mechanism in horizontal into simulation because the elevating mechanism's performance is similar to the traversing mechanism in this study.

The traversing mechanism is usually fixed under the gun tower. Its output gears and the transmission mechanism are shown in Fig.4 which shows that the traversing mechanism consists of a multiple gear system. The gears are led by the motor to revolve to drive the gear ring revolve.

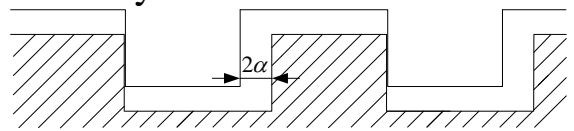

Fig.4 the structure of gear gap

According to the relationship between gear's moving position and the torque, the equation of the gear's transmission mechanism is shown as fellow.

$$
\tau(\Delta \theta)= \begin{cases}k_{\tau}(\Delta \theta-\alpha) & \Delta \theta>\alpha \\ 0 & |\Delta \theta| \leq \alpha \\ k_{\tau}(\Delta \theta+\alpha) & \Delta \theta<-\alpha\end{cases}
$$

In this equation, the $\tau(\Delta \theta)$ is the output torque, the $k_{\tau}$ is the stiffness coefficient, $\Delta \theta=\theta-\theta_{m}$ is the displacement of tooth gap, $2 \alpha$ is the width of the tooth gap. With an increasingly wearing, the width of the gap will get bigger and bigger.

\section{Simulation}

In order to simulate the gear of traversing mechanism, the relative position of main gear in traversing mechanism and the evolvent of gear ring. The width of the gap between the gear and the 
ring is set to be $d=0 \mathrm{~mm}, 0.5 \mathrm{~mm}, 1 \mathrm{~mm}, 1.5 \mathrm{~mm}, 2 \mathrm{~mm}, 2.5 \mathrm{~mm}$. And set the velocity of the gear as $v=\sin (t) m \cdot s^{-1}$. In different width of gap, begin to simulate the gun tower system, and the different impacts were shown as Fig.5.

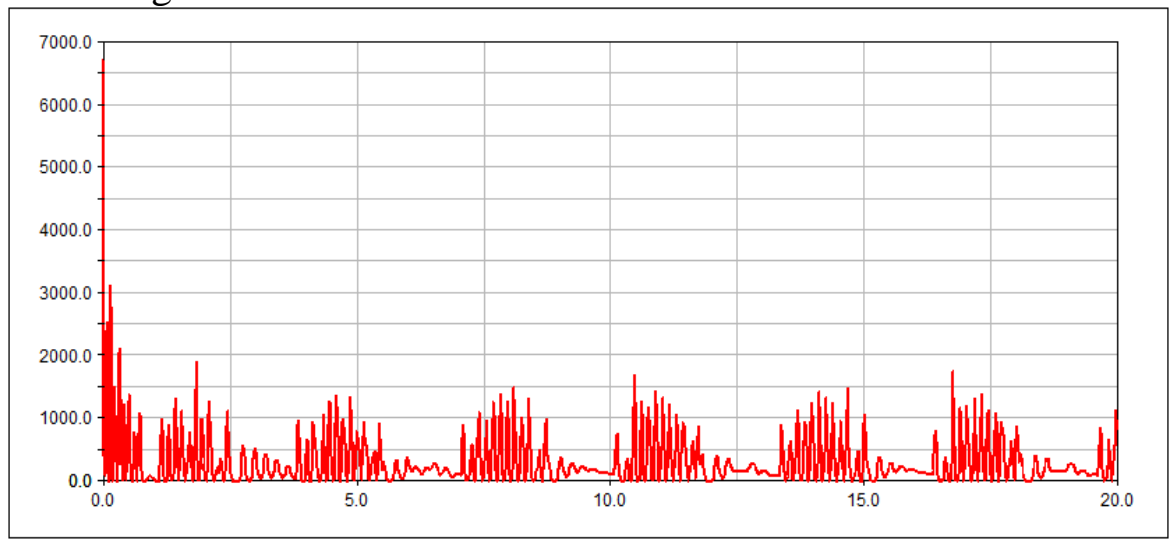

(a) $d=0 \mathrm{~mm}$

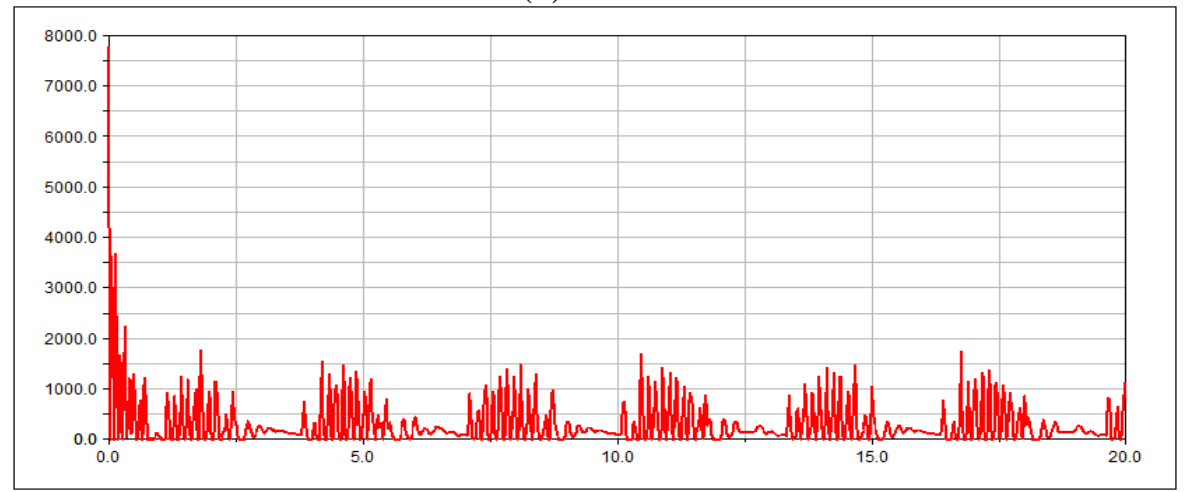

(b) $d=1 \mathrm{~mm}$

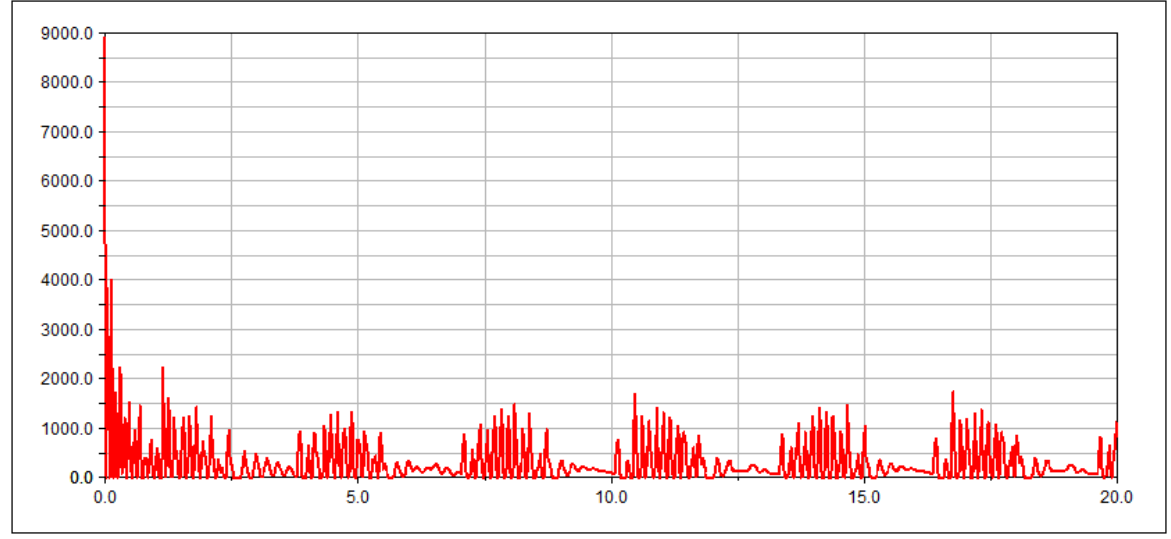

(c) $d=2 \mathrm{~mm}$

Fig.5 the impacts in different gaps

From the Fig.5, it can be found that with the gap getting wider and wider, the impact of the gear of the traversing mechanism has an increasingly development. It can also fasten the gear's deformation, also including the gear ring, the gun towerand the traversing mechanism. In real life, the gap must be controlled in a fit distance so that the gun tower system can be ensured a long used life.

\section{Conclusion}

Firstly, a 3D gun solid model and its simulation is established after an introduction about the transmission in both the traversing mechanism and the elevating mechanism. It is analyzed that the impact will increase when the gear gap become bigger and bigger. And it is also proved that the gear gap is not a linear factor in transmission structure, however, it has a huge influence on it. In real life, the gear gap must be controlled. 


\section{References}

[1] ZANG Ke-mao, LI Li-yu, LI Kuang-cheng. A Study on A.C. All-electric Tank Gun Control System[J]. ActaArmamentarii, 2006,27(3):549-552.

[2] YAN Zhi-feng, MA Xiao-jun, ZANG Ke-mao. Soft Switching Boost Converter Used in a Novel Tank Gun Control System[J]. Transactions of China Electro technical Society,2007,22(10):128-132.

[3] R.DeDoncker and J.P.Lyons, The Auxiliary Resonant Commutated Pole Converter[C]. Conf.Rec. IEEE-IAS Annu. Meeting, 1990, p1128-1135.

[4] OlorunfemiOjo, Innocent Ewean Davidson. PWM-VSI Inverter-assisted Stand-alonedual Stator Winding Induction Generator.[J].IEEE Trans.On.IA,2003,36(6):1604-1611.

[5] Wan Xiaopeng, Hou Chi, Zhao Meiying.Development of Auto-Modeling Program Based on ADAMS Marcos for Aircraft Landing gear Dynamic Loads Simulation Analysis.[J].Second International Conference on Intelligent Computation Technology and Automation,2009,p23-26.

[6] Zhu Dalin, Qin Jinyi, Zhang Yi. Research on Co-simulation Using ADAMS and MTALAB for Active Vibration Isolation System.[J].International Conference on Intelligent Computation Technology and Automation,2010,p1126-1129. 